\title{
CARDIAC MAGNETIC RESONANCE ASSESSMENT OF PROGRESSIVE MYO- PERICARDITIS DUE TO COBALT CARDIOTOXICITY
}

Massimiliano Lorenzini ${ }^{1,2}$, Mohammed Y. Khanji ${ }^{1,3}$, Luis Rocha Lopes ${ }^{1,2}$, Charlotte Manisty $^{1,2}$, Konstantinos Savvatis ${ }^{1,2,3}$.

${ }^{1}$ Department of Cardiovascular Imaging, Barts Heart Centre, St. Bartholomew’s Hospital, London, UK;

${ }^{2}$ University College London Institute of Cardiovascular Science, London, UK;

${ }^{3}$ William Harvey Research Institute, NIHR Barts Biomedical Research Centre, Queen Mary University London, London, UK.

Conflicts of Interest: Nothing to Disclose.

Corresponding author: Dr. Massimiliano Lorenzini

Inherited Cardiovascular Diseases, Barts Heart Centre

St Bartholomew’s Hospital

W Smithfield, EC1A 7BE

London, United Kingdom

dr.m.lorenzini@gmail.com 
A 48-year-old man was referred with a 3-year history of recurrent pericardial effusion requiring drainage, and progressive heart failure. Past medical history was unremarkable other than bilateral hip replacement with metal-on-metal implants for arthritis at 39-years of age.

ECG showed low voltages (panel B). Bloods, including an extensive rheumatology work up, were unremarkable apart from persistently mildly elevated troponin T (40ng/l, normal $<14 \mathrm{ng} / \mathrm{l})$. Initial cardiac magnetic resonance (CMR) showed biventricular dysfunction with subepicardial infero-lateral and midwall septal late enhancement (LGE, panel A). FDG-PET showed mild diffuse cardiac uptake and corresponding to the hip prostheses (panel $\mathbf{H}$ ). Endomyocardial biopsy showed myocyte hypertrophy, focal fibrosis and endocardial thickening with no evidence of active myocarditis or amyloid. Repeat CMR at 15 months showed chronic pericardial effusion, a now severe biventricular dysfunction (Panel C, Supplementary Video 1), mildly raised native T2 (55ms @ 1.5T, normal 40-51ms, panel D) and native T1 (1080ms, normal 970-1050ms, panel E), left ventricular apical thrombus on early gadolinium enhancement (panel F, arrow) and a striking progression of LGE (panel G). Myocardial T2* was normal (45ms) and liver T2* was mildly reduced (10ms).

Serum chromium and cobalt levels were extremely elevated $[1279 \mathrm{nmol} / \mathrm{L}$ (normal $<134$ $\mathrm{nmol} / \mathrm{L}$ ) and $5647 \mathrm{nmol} / \mathrm{L}$ (normal $<118 \mathrm{nmol} / \mathrm{L}$ ), respectively] and subsequent mass spectrometry of the myocardial biopsy confirmed very high myocardial levels $[17.2 \mathrm{ug} / \mathrm{g}$ (normal $<0.6 \mathrm{ug} / \mathrm{g}$ ) and $4.7 \mathrm{ug} / \mathrm{g}$ (normal $<0.5 \mathrm{ug} / \mathrm{g}$ ), respectively].

CMR can demonstrate the typical features of this rare but recognised complication of metalon-metal hip prostheses, in this case with a striking progression of LGE. 


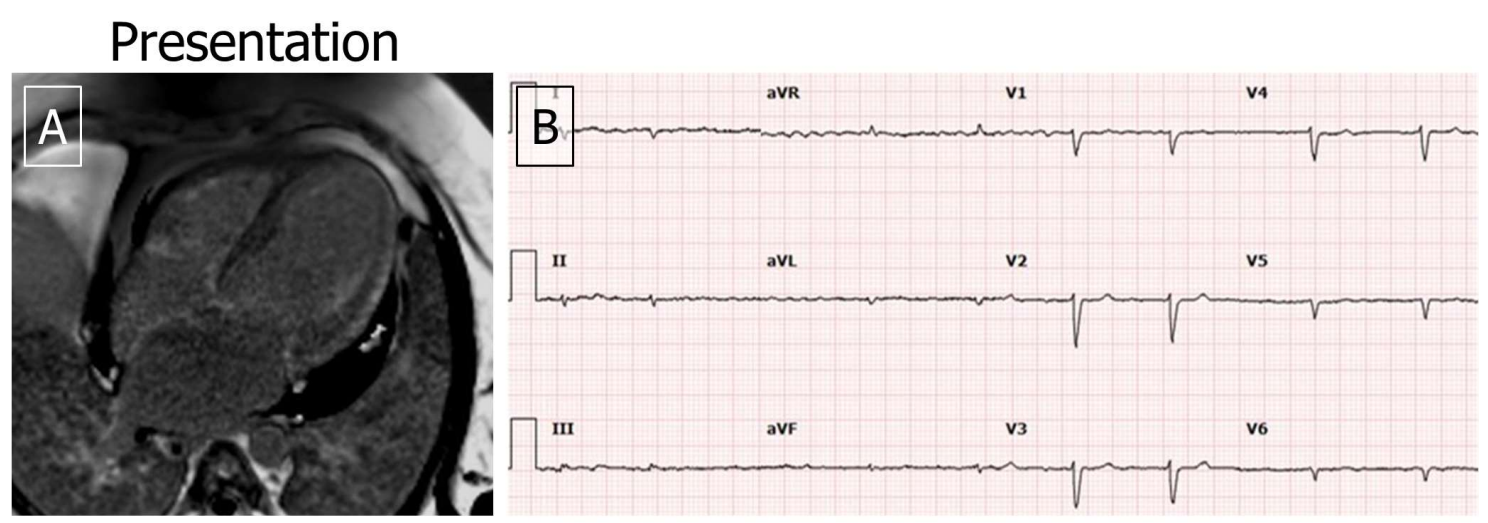

15 months follow up
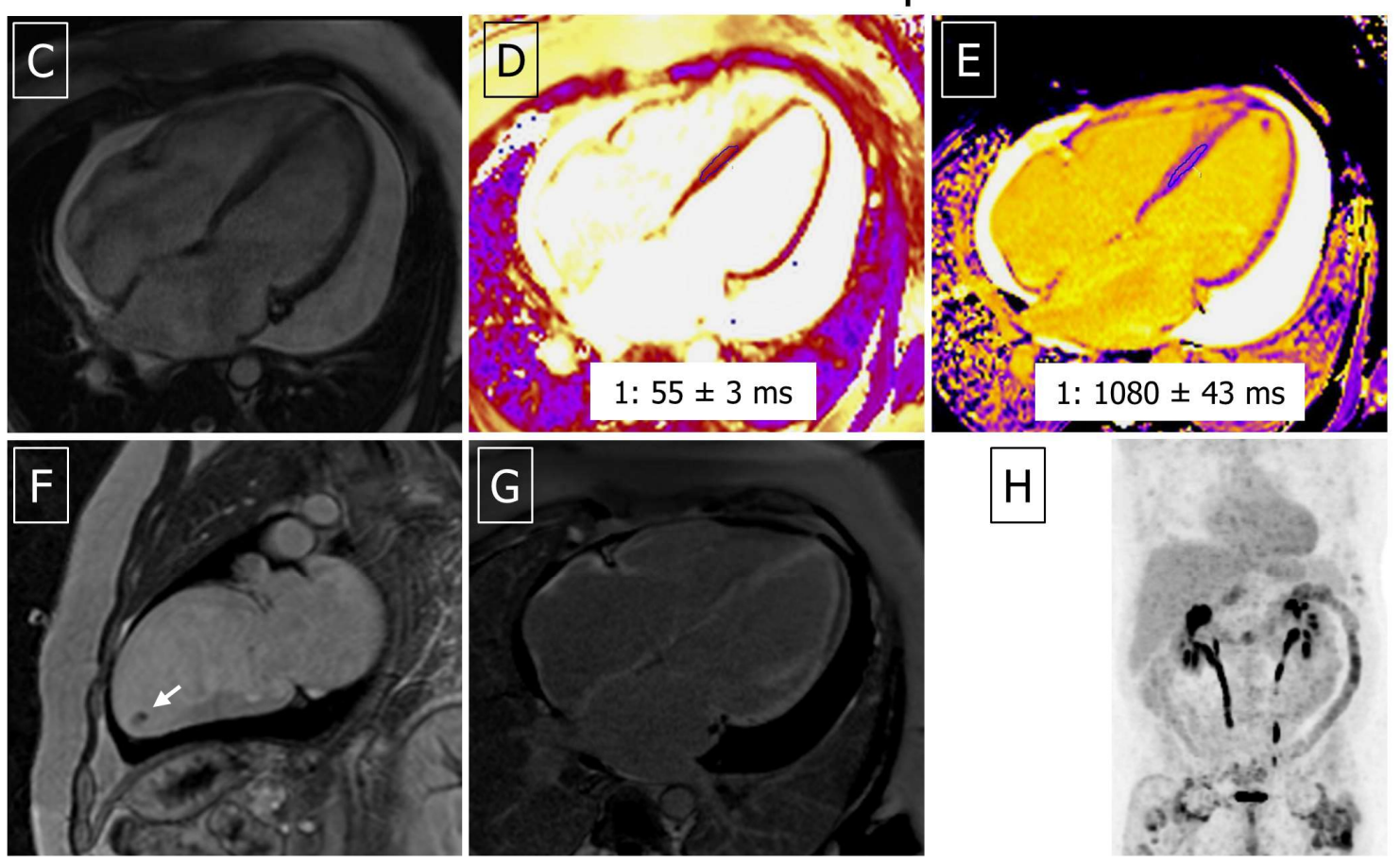

$\mathrm{H}$

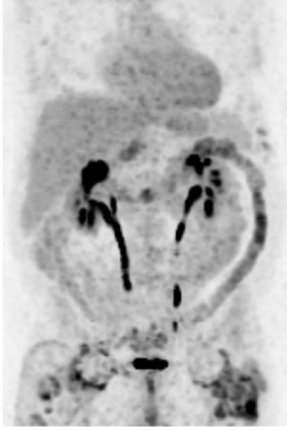

